\title{
Variabilidad espacial y estacional de temperatura, salinidad y densidad en Bahía Concepción, Golfo de California, México
}

Spatial and seasonal variability of temperature, salinity and density in Bahía Concepción, Gulf of California, Mexico

\author{
Maclovio Obeso-Nieblas ${ }^{1}$, Juan Heberto Gaviño-Rodríguez ${ }^{2}$ e Hipolyto Obeso-Huerta ${ }^{3}$ \\ ${ }^{1}$ Centro Interdisciplinario de Ciencias Marinas, Instituto Politécnico Nacional, Ave. Inst. Politécnico Nacional S/N, Col. \\ Playa Palo de Santa Rita, La Paz B.C.S. 23090, México, mniebla@ipn.mx \\ ${ }^{2}$ Instituto Oceanográfico de la Universidad de Colima, Kilómetro 20, Carretera Manzanillo, Cihuatlán, Manzanillo Colima \\ 28860, México \\ ${ }^{3}$ Instituto Tecnológico de La Paz, Boulevard Forjadores de Baja California Sur No.4720, La Paz, B.C.S. 23080, México
}

\begin{abstract}
This study analyzed the seasonal and spatial (longitudinal, transverse and depth) variability of temperature, salinity, and density in the Bahía Concepción in the Gulf of California, Mexico. CTD data from 4 oceanographic cruises carried out during 1994 (winter, spring, summer, and autumn) were used. The presence of wind-driven southeast upwelling at the mouth of the Bahía de Concepción was found during stratified and warm conditions in the spring and summer of 1994, with the highest index of wind-induced upwelling and the maximum frequency of Brunt-Väisälä in spring. The Bay was stratified and warm in temperature during spring and summer with the thermocline, halocline, and pycnocline more pronounced in spring. This was mainly due to increased solar radiation and the arrival of cold and less salty water from the Gulf of California, caused by upwelling on the west coast of the gulf generated by the winds from the southeast. Meanwhile in autumn and winter the Bahía Concepción showed homogeneous and cold conditions, produced by strong northwest winds and lower solar radiation. The distribution of temperature, salinity, and density varied seasonally and spatially due to solar radiation, warm stratification, upwelling, and wind-driven flow. Conditions in the Bay ranged from homogeneous and cold to stratified and warm, apparently separated by two short transition periods.
\end{abstract}

Key words: Thermocline, wind, radiation, upwelling, stratification

\begin{abstract}
Resumen.- Se analizó la variabilidad estacional y espacial (longitudinal, transversal y profundidad) de la temperatura, salinidad y densidad en Bahía Concepción en Golfo de California, México, con datos de CTD de 4 cruceros oceanográficos realizados durante 1994 en invierno, primavera, verano y otoño. Se encontró la presencia de la surgencia impulsada por el viento del sureste en la boca de Bahía Concepción durante la condición estratificada y cálida de primavera y verano de 1994, con el mayor índice de surgencia inducida por el viento y la máxima frecuencia de Brunt-Väisälä en primavera. Se observó la bahía estratificada y cálida durante primavera y verano, con la termoclina, haloclina y picnoclina más pronunciadas en primavera, debido principalmente a la mayor radiación solar recibida y al arribo de aguas frías y menos saladas desde el Golfo de California, originadas por la acción de la surgencia en la costa occidental del Golfo de California, generada por los vientos del sureste. Mientras tanto, en otoño e invierno se presentó la condición homogénea y fría, producida por los fuertes vientos del noroeste y la menor radiación solar recibida. La distribución de temperatura, salinidad y densidad difieren estacional y espacialmente, producto de la radiación solar, estratificación por calentamiento, la surgencia en la boca de la bahía y el flujo impulsado por el viento, fluctuando de una condición homogénea y fría a un ambiente estratificado y cálido, separado al parecer por dos períodos cortos de transición.
\end{abstract}

Palabras clave: Termoclina, viento, radiación, surgencia, estratificación

\section{INTRODUCCIÓN}

Las propiedades físicas del agua de mar son importantes para una gran variedad de procesos oceánicos. Algunas de estas propiedades son la temperatura, salinidad, densidad, conductividad, entre otras. Las variables físicas de mayor importancia para los oceanógrafos son la temperatura, salinidad y densidad. Son variables que cambian en el espacio y en el tiempo, producen gradientes horizontales y verticales que modifican los patrones de circulación, mismos que a su vez se relacionan con las condiciones morfológicas de las lagunas costeras, las profundidades, aportes de flujos externos (mareas, aportes fluviales y subterráneos) y la acción de los vientos. La 
combinación de estas características permite mayor o menor penetración de luz hacia el fondo de la laguna, situación que favorece o inhibe la productividad primaria (Obeso-Nieblas 2003).

Bahía Concepción se caracteriza por ser un cuerpo de agua somero con un canal localizado en su porción noroeste. Es una región con una importante perspectiva turística y pesquera, lo que representa un importante capital ecológico para el desarrollo económico del Estado de Baja California Sur. En este contexto, la conservación de los ecosistemas marinos queda eslabonada con el principio de desarrollo sustentable (Obeso-Nieblas et al. 1996).

Su ubicación geográfica y alta productividad biológica la convierten en uno de los cuerpos costeros más significativos de la parte central del Golfo de California dado que en ella existe una amplia biodiversidad de especies marinas (Mateo-Cid et al. 1993, Villalejo-Fuerte \& Ochoa-Báez 1993, Martínez-López \& Gárate-Lizárraga 1994; Rodríguez-Romero et al. 1994, Palomares-García et al. 2002). Además, las condiciones oceanográficas en la región modulan cambios estaciónales en la productividad de la bahía (Gilmartin \& Revelante 1978, Lechuga-Deveze \& Morquecho-Escamilla 1998).

La estratificación en Bahía de Concepción está relacionada con la transferencia de calor estacional de la atmósfera, así como a las intrusiones de agua fría forzadas por la surgencia impulsada por el viento. Durante invierno, la columna de agua está relativamente bien mezclada por enfriamiento de la atmósfera y por los vientos del noroeste que suelen superar los $10 \mathrm{~m} \mathrm{~s}^{-1}$, que favorecen hundimientos. Durante el verano, la columna de agua se calienta gradualmente y se estratifica por el flujo de calor de la atmósfera. El campo del viento cambia de favorecer el hundimiento a favorecer la surgencia a principios de verano, es decir, los vientos se tornan predominantemente del sureste (Cheng et al. 2010).

El patrón de vientos estacional es predominantemente del noroeste durante otoño e invierno (período frío) y del sureste durante primavera y verano (período cálido). Los vientos del noroeste producen una columna de agua bien mezclada durante otoño e invierno, y la disminución de estos vientos inicia el período de transición de una columna bien mezclada y fría a condiciones cálidas y estratificadas (Palomares-García et al. 2006).

La región de Bahía Concepción y sus alrededores presentan clima tipo BW (desértico). Esta bahía tiene características anti-estuarinas, y la temperatura superficial promedio anual es $24,05^{\circ} \mathrm{C}$, con una mínima de $17,50^{\circ} \mathrm{C}$ (enero) y una máxima de $30,60^{\circ} \mathrm{C}$ (septiembre). La salinidad tiene un promedio anual de 35,30, con un mínima de 34,60 y máxima y 37,00 (Félix-Pico 1987).

La marea en Bahía Concepción tiene un comportamiento mixto semidiurno, con la secuencia pleamar superior bajamar inferior, lo que produce corrientes de reflujo más intensas que las de flujo; esta característica permite que los canales de la bahía permanezcan dragados (ObesoNieblas et al. 1996).

La relevancia de este trabajo radica en ser el primer estudio que incluye un número importante de datos oceanográficos de CTD, registrados estacionalmente durante el año 1994, en todo lo largo, ancho y profundo de la bahía. En la parte central de la bahía análisis previos, principalmente enfocados en estudios biológicos, han sido realizados únicamente con datos de temperatura y excepcionalmente de salinidad, y a pesar de esta condición, se han formulado conclusiones para todo el cuerpo de agua. En el planteamiento y desarrollo de esta investigación durante 1994, se supuso que la variación espacial y temporal de temperatura, salinidad y densidad en Bahía Concepción está determinada principalmente por la radiación solar, la acción de los vientos y el flujo impulsado por el gradiente de densidad. El objetivo del presente estudio consistió en analizar la variabilidad temporal (estacional) y espacial (longitudinal, transversal y profundidad) de temperatura, salinidad y densidad en Bahía Concepción, durante el año 1994.

\section{Materiales y Métodos}

\section{ÁREA DE ESTUdio}

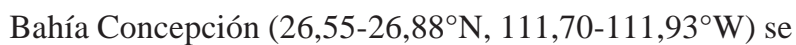
ubica en la parte central de la costa este de la Península de Baja California México, tiene una extensión de 40 km de largo por 4,5 km de ancho en su parte más angosta y 9,5 km en la parte más ancha. Se sitúa en dirección paralela al Golfo de California con el cual se comunica en su parte norte (Obeso-Nieblas et al. 1996). La bahía se caracteriza por ser un cuerpo de agua somero con un canal de $30 \mathrm{~m}$ de profundidad localizado en la porción noroeste, contiguo a la costa de forma ovalada en dirección norte sur (Fig. 1). 


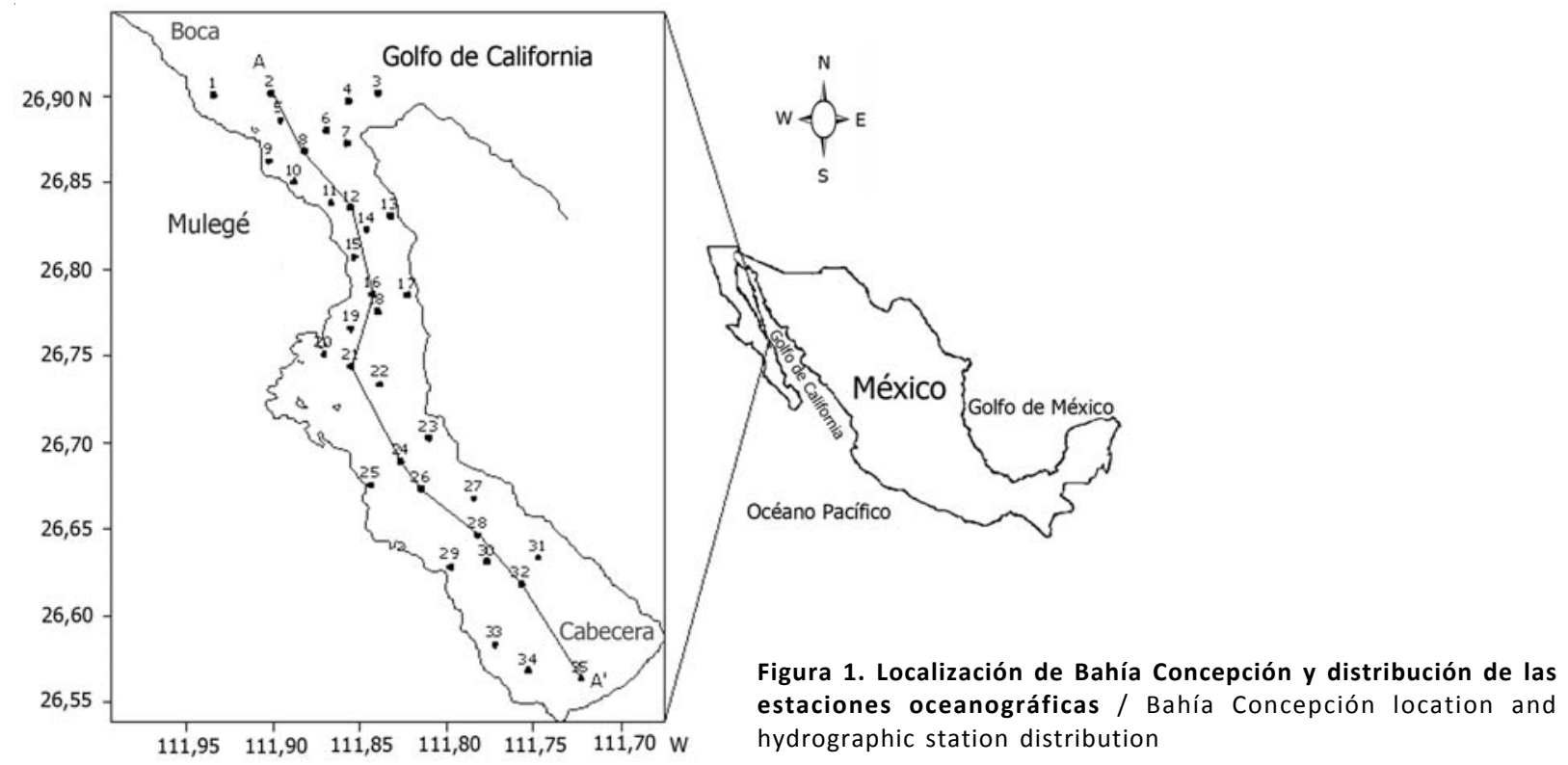

\section{Trabajo en TERRENo}

Los datos hidrográficos fueron obtenidos con un CTD marca InterOcean modelo S4, durante 4 muestreos realizados del 24 al 25 de febrero, del 20 al 21 de junio, del 7 al 9 de noviembre, y del 19 al 21 de septiembre de 1994, a bordo de una embarcación tipo yate, CICIMAR-XV (Fig. 1). Se obtuvieron registros de conductividad $\left(\mathrm{mS} \mathrm{cm}^{-1}\right)$, profundidad (m) y temperatura $\left({ }^{\circ} \mathrm{C}\right)$. La salinidad y la densidad fueron calculadas de acuerdo a los procedimientos estándares (UNESCO 1991), según una red de muestreo que contiene 35 estaciones. Los datos de salinidad fueron corroborados con el análisis de muestras en el laboratorio a profundidades estándares.

Con la finalidad de responder a la hipótesis planteada y cumplir con el objetivo de la investigación, se realizaron perfiles verticales promedio de temperatura, salinidad y densidad, así como la distribución superficial de temperatura y salinidad. Además, se efectuó la distribución vertical de temperatura, salinidad y densidad en una sección longitudinal desde la boca frontera con el Golfo de California hasta la cabecera de la bahía (sección A-A', estaciones 2 a 35) y transversales en 3 secciones (boca, parte central y la cabecera de la bahía) (Fig. 1). La velocidad del viento, la radiación solar y evaporación fueron obtenidas en el Servicio Meteorológico Nacional de México y en la Estación Mulegé de la Comisión Nacional del Agua, respectivamente.
La cuantificación de la estratificación de la columna de agua se determinó a partir del cálculo del parámetro de estratificación $\Phi$ (Joules por metro cúbico, J m-3) (Simpson 1981), desde la superficie hasta los $30 \mathrm{~m}$ de profundidad, de acuerdo a la siguiente ecuación:

$$
\phi=1 / h \int_{-h}^{0}(\rho(z)-\rho) g z d z
$$

donde $h$ es la profundidad, $z$ la coordenada vertical (positiva hacia la superficie), $\rho$ la densidad, $\rho$ la densidad promedio y $g$ la aceleración de la gravedad.

Se evaluaron los principales mecanismos de estratificación y mezcla en la bahía durante los 4 muestreos de 1994, mediante los siguientes forzamientos, según Simpson et al. (1990) y Cheng et al. (2010):

a) Estratificación por calentamiento

$$
\frac{d \phi}{d t}=\frac{\alpha g Q}{2 C_{p}}
$$

donde $\alpha$ es el coeficiente de expansión térmica del agua de mar $\left(\sim 1,610^{-4}{ }^{\circ} \mathrm{C}^{-1}\right)$, g es la aceleración de la gravedad $\left(9,81 \mathrm{~ms}^{-2}\right)$, $\mathrm{Q}$ es la radiación recibida del sol (Watts $\left.\mathrm{m}^{-2}\right)$ y $\mathrm{C}_{\mathrm{p}}$ es el calor especifico del agua de $\operatorname{mar}\left(\sim 4,010^{3} \mathrm{~J} \mathrm{Kg}^{-1}\right.$ ${ }^{\circ} \mathrm{C}^{-1}$ ) 
b) Estratificación por flujo de densidad

$$
\frac{d \phi}{d t}=\frac{1}{320} \frac{g^{2} H^{4}}{A_{v} \rho}\left(\frac{\partial \rho}{\partial x}\right)^{2}
$$

donde $A_{v}\left(1,310^{-4} \mathrm{~m}^{2} \mathrm{~s}^{-1}\right)$ es el coeficiente de viscosidad vertical turbulento, se estimó según la aproximación de Pacanowski \& Philander (1981), $\rho$ es la densidad del agua de mar $\left(\mathrm{kg} \mathrm{m}^{-3}\right), H$ es la profundidad en $\mathrm{m}$ y $\left(\frac{\partial \rho}{\partial x}\right)$ es el gradiente horizontal de densidad $\left(\mathrm{kg} \mathrm{m}^{-3} \mathrm{~m}^{-1}\right)$, con el eje $\mathrm{x}$ orientado a lo largo de la bahía de la boca a la cabecera.

c) Estratificación por esfuerzo de marea

$$
\frac{d \phi}{d t}=0,031 g H \frac{\partial \rho}{\partial x} \hat{u}
$$

donde û $\left(\mathrm{m} \mathrm{s}^{-1}\right)$ es la magnitud promedio del perfil de corrientes

d) Estratificación por flujo impulsado por el viento.

$$
\frac{d \phi}{d t}=\frac{1}{48} \frac{g H^{2} \tau}{A_{v} \rho} \frac{\partial \rho}{\partial x}
$$

donde $\tau$ es el esfuerzo del viento $\left(\mathrm{N} \mathrm{m}^{-2}\right)$, cuando el esfuerzo del viento y el gradiente horizontal de densidad $\left(\frac{\partial \rho}{\partial x}\right)$ tienen el mismo signo, el flujo impulsado por el viento aumenta la estratificación, mientras que, cuando $\tau$ y $\frac{\partial \rho}{\partial x}$ tienen signos opuestos, el flujo impulsado por el viento disminuye la estratificación (Cheng et al. 2010).

e) Mezcla por velocidad del viento

$$
\frac{d \phi}{d t}=-\delta C_{d s} \rho_{a} \frac{U_{w}^{3}}{H}
$$

La velocidad del viento $\left(U_{w} \mathrm{~m} \mathrm{~s}^{-1}\right)$ a $10 \mathrm{~m}$ sobre la superficie, disminuye la estratificación, donde $C_{d s}(6,4$ $10^{-5}$ ) es el coeficiente efectivo del esfuerzo de arrastre para la superficie, $\delta(0,023)$ es el coeficiente de eficiencia de mezcla, $\rho_{a}$ es la densidad del aire $\left(1,3 \mathrm{~kg} \mathrm{~m}^{-3}\right)$

f) Mezcla por corrientes de marea

$$
\frac{d \phi}{d t}=-\frac{4}{3 \pi} \varepsilon C_{d} \rho \frac{U_{T}{ }^{3}}{H}
$$

La velocidad de las corrientes de marea $\left(U_{T} \mathrm{~m} \mathrm{~s}^{-1}\right)$ disminuyen la estratificación, donde $\varepsilon(0,0037)$ es el coeficiente de eficiencia de mezcla y $\mathrm{C}_{d}(0,0025)$ es el coeficiente del esfuerzo de arrastre en el fondo.

Se calculó el Índice de Surgencia costera inducida por el viento expresado en $\left(\mathrm{m}^{3} \mathrm{~s}^{-1}\right)$ por $100 \mathrm{~m}$ de costa, para los vientos (sureste) que soplan paralelos a la costa y que tienen posibilidad de generar una surgencia costera en la región de la boca de Bahía Concepción, mediante la siguiente ecuación (Silva \& Valdenegro 2003).

$$
I S C=\frac{\rho_{a} C_{d}|\vec{V}| V_{y}}{f \rho}
$$

donde $|\vec{V}|$ es la magnitud promedio del viento en $\mathrm{m} \mathrm{s}^{-1}, \rho_{a}$ es la densidad del aire, $\rho$ es la densidad del agua de mar, $f$ es el parámetro de Coriolis, $V_{y}$ es la componente del viento a lo largo de la costa y $C_{d}$ es el coeficiente de arrastre del viento en la superficie.

También se calculó la frecuencia de Brunt-Väisälä $(N)$ para cuantificar la importancia de la estratificación, esta variable se considera fundamental en la dinámica de fluidos estratificados. Puede ser interpretada como la frecuencia vertical excitada por un desplazamiento vertical de la parcela de fluido, se obtiene por la expresión siguiente (Plata \& Filonov 2007):

$$
N=\sqrt{\frac{g \partial \rho}{\bar{\rho} \partial z}}
$$

donde $N$ es la frecuencia de Brunt-Väisälä $\left(\mathrm{s}^{-1}\right), g$ es la aceleración de la gravedad, $\rho$ es la densidad promedio de la parcela de agua desplazada y $\frac{\partial \rho}{\partial x}$ es el gradiente vertical de densidad $\left(\mathrm{kg} \mathrm{m}^{-3} \mathrm{~m}^{-1}\right)$.

\section{Resultados}

Los resultados se presentan divididos en 2 categorías: la condición estratificada y cálida, así como la condición homogénea y fría en la bahía, durante los 4 muestreos estacionales de 1994.

Durante primavera (junio) y verano (septiembre), se presentó la condición estratificada y cálida. En primavera, se apreció la bahía cálida con una temperatura superficial promedio de $27,89^{\circ} \mathrm{C}$ y con una capa de mezcla mínima (1$5 \mathrm{~m}$ ) en el interior de la bahía. Destacó la presencia de una termoclina intensa, desde la superficie (en algunas estaciones) hasta los $15 \mathrm{~m}$ de profundidad, con un gradiente vertical de $0,64^{\circ} \mathrm{C} \mathrm{m}^{-1}$ (Fig. 2a). En la zona de la boca se presentó un gradiente horizontal superficial de $4,7710^{-40} \mathrm{C} \mathrm{m}^{-1}$ (Fig. 3a).

En la sección longitudinal entre la boca y la cabecera de la bahía, la isoterma de los $19,0^{\circ} \mathrm{C}$ marcó el fondo de la termoclina estacional, con la mayor estratificación entre los 3 y $10 \mathrm{~m}$ de profundidad, con un parámetro de estratificación $(\phi)$ promedio de 35,39 J m³ . Se observaron 

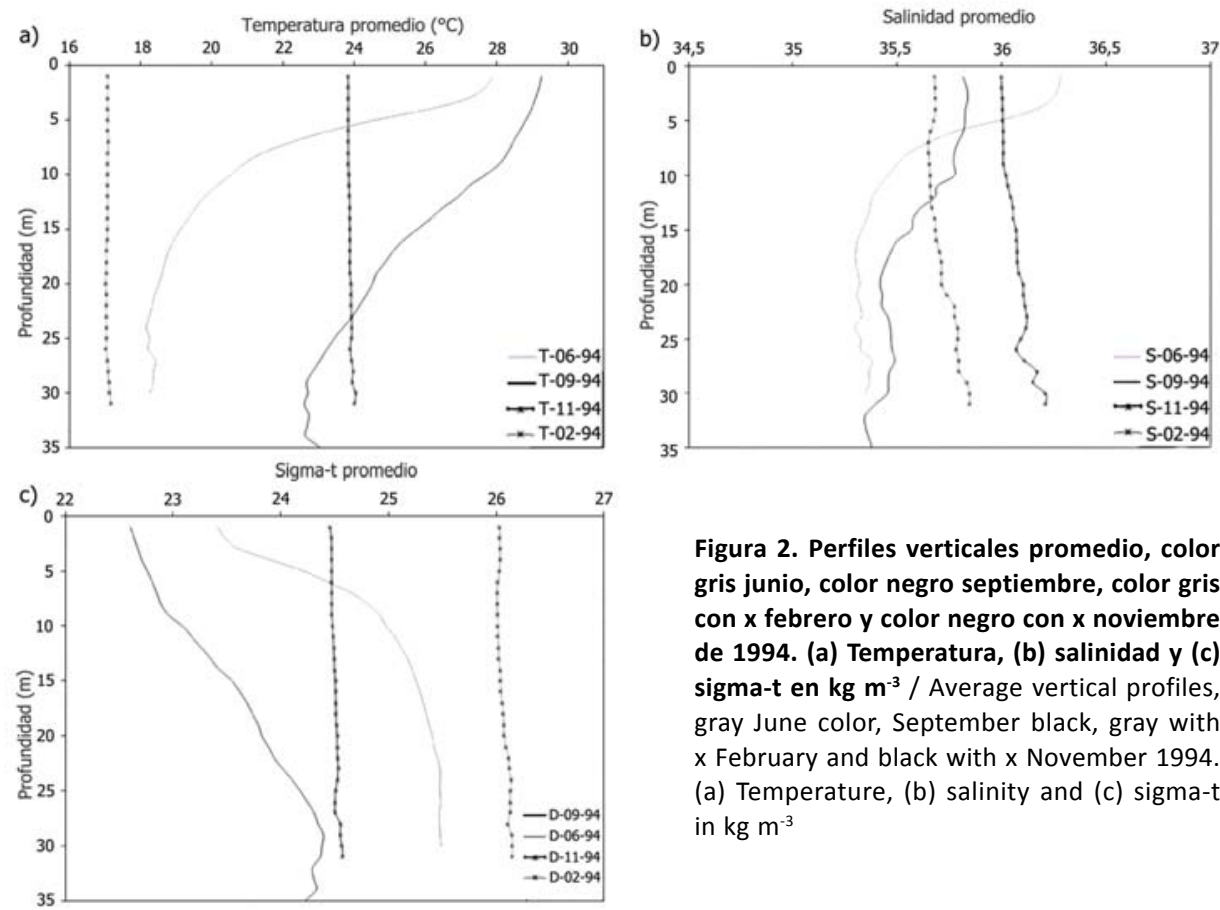

Figura 2. Perfiles verticales promedio, color gris junio, color negro septiembre, color gris con $x$ febrero y color negro con $x$ noviembre de 1994. (a) Temperatura, (b) salinidad y (c) sigma-t en $\mathbf{~ k g ~ m}^{-3}$ / Average vertical profiles, gray June color, September black, gray with x February and black with x November 1994. (a) Temperature, (b) salinity and (c) sigma-t in $\mathrm{kg} \mathrm{m}^{-3}$

a)

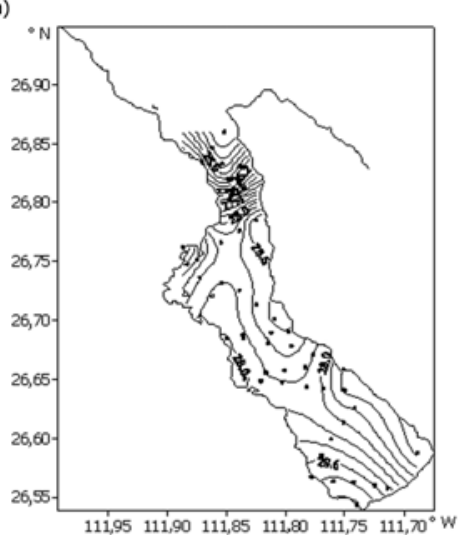

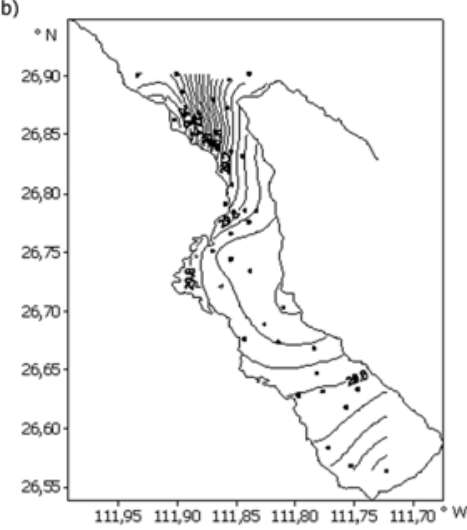
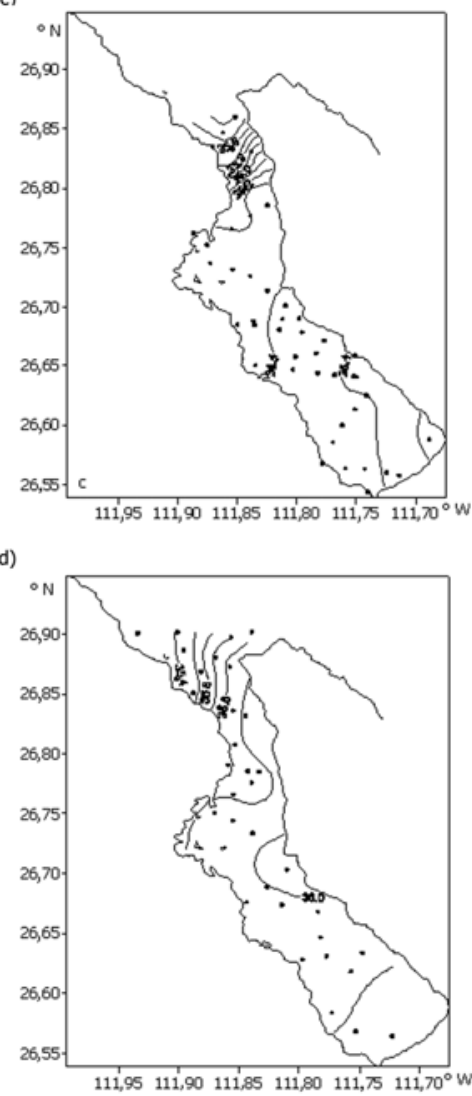

Figura 3. Distribución horizontal superficial de 1994. (a) Temperatura, junio, (b) temperatura, septiembre, (c) salinidad, junio y (d) salinidad, septiembre / Horizontal distribution of surface 1994. (a) Temperature, June, (b) temperature, September, (c) salinity, June and (d) salinity, September 
las aguas de la boca más frías $\left(25,0^{\circ} \mathrm{C}\right)$, que en la cabecera $\left(28,0^{\circ} \mathrm{C}\right)$. Se apreció en la boca y la zona adyacente la elevación y el afloramiento de las isotermas de 25,0 a $28,0^{\circ} \mathrm{C}$ entre las estaciones S2 y S16 (Fig. 4a). El parámetro de estratificación $(\phi)$ cambió espacialmente en la bahía: en la boca fue de 26,58 $\mathrm{J} \mathrm{m}^{-3}$ (estación S2), se incrementó hacia la región central hasta 50,09 $\mathrm{J} \mathrm{m}^{-3}$ (estación S21) y disminuyó hacia la cabecera 33,17 J m³ (estación S28).

La distribución transversal de temperatura en primavera presentó el siguiente comportamiento: en la sección de la boca estuvo más cálida la costa Oeste, con el afloramiento de la isotermas de los 25,0 y 26, $0^{\circ} \mathrm{C}$ (Fig. 5a). En la sección media la costa Este fue la más cálida y ocurrió el afloramiento de la isoterma de los $28^{\circ} \mathrm{C}$ en la costa Oeste (Fig. 5b). Mientras que en la sección de la cabeza la costa Oeste fue la más cálida con el afloramiento de la isoterma de los $28^{\circ} \mathrm{C}$ en la costa Este (Fig. 5c). La radiación solar promedio para este mes (junio) fue de $350 \mathrm{~W} \mathrm{~m}^{-2}$, con una evaporación de $300 \mathrm{~mm}$ y los vientos fueron débiles provenientes del sureste de $4,5 \mathrm{~m} \mathrm{~s}^{-1}$, con una frecuencia de Brunt-Väisälä promedio de $3,410^{-2} \mathrm{~s}^{-1}$, con el valor máximo a los $6 \mathrm{~m}$ de profundidad $\left(4,4510^{-2} \mathrm{~s}^{-1}\right)$ y un índice de surgencia costera inducida por el viento de $54,7 \mathrm{~m}^{3} \mathrm{~s}^{-1}$ (Tabla 1).

La salinidad en primavera registró el promedio superficial máximo de 36,28 disminuyendo rápidamente hacia el fondo, dando origen a una estratificación intensa.
La haloclina se presentó desde la superficie en ciertas estaciones hasta los $10 \mathrm{~m}$ de profundidad, con un gradiente vertical de $0,09 \mathrm{~m}^{-1}$ (Fig. 2b). En la zona de la boca se registró un gradiente horizontal superficial de $1,3710^{-4} \mathrm{~m}^{-1}$ (Fig. 3c). Longitudinalmente, se aprecia una columna estratificada en los primeros $10 \mathrm{~m}$ y una capa de mezcla mínima en la parte central y la cabecera, con la isohalina de las 35,4 marcando el fondo de la haloclina. La salinidad se incrementó desde la boca $(35,4)$, hacia la cabecera $(36,4)$, con el afloramiento de las isohalinas de 35,4 a 36,4 en la boca y la parte central, mientras que en la cabecera se localizaron hasta los $13 \mathrm{~m}$ de profundidad (Fig. 6a).

La distribución transversal de salinidad en la bahía mostró las siguientes características: en la sección de la boca la costa Oeste tuvo la mayor salinidad, con el afloramiento de las isohalinas de 35,4 a 35,6, en esta sección no se registró estratificación (Fig. 7a). En la sección media de la bahía se encontró una estratificación horizontal con la menor salinidad en la parte intermedia de la sección $(36,3)$, con el afloramiento de la isohalina de 36,4 enfrente de ambas costas (Fig. 7b). Mientras que en la sección de la cabeza la costa Este presentó la mayor salinidad, con el afloramiento de la isohalina de 36,4 en la costa Oeste, con estratificación horizontal entre los 3 y $11 \mathrm{~m}$ de profundidad (Fig. 7c).

Tabla 1. Comparación de valores de variables físicas, de la columna de agua, para febrero, junio, septiembre y noviembre de 1994, en Bahía Concepción, México / Comparison of values of physical variables of the water column, to February, June, September and November 1994 in Bahía Concepción, Mexico

\begin{tabular}{|c|c|c|c|c|}
\hline Variable & Febrero & Junio & Septiembre & Noviembre \\
\hline Tem.sup.prom. $\left({ }^{\circ} \mathrm{C}\right)$ & $17,07 \pm 0,15$ & $27,89 \pm 1,13$ & $29,25 \pm 1,27$ & $23,82 \pm 0,24$ \\
\hline Sal.sup.prom.(ups) & $35,67 \pm 0,17$ & $36,28 \pm 0,30$ & $35,81 \pm 0,22$ & $35,99 \pm 0,26$ \\
\hline Sigma-t sup.prom. $\left(\mathrm{kg} \mathrm{m}^{-3}\right)$ & $26,03 \pm 0,11$ & $23,41 \pm 0,18$ & $22,60 \pm 0,27$ & $24,45 \pm 0,15$ \\
\hline Velocidad del viento $\left(\mathrm{m} \mathrm{s}^{-1}\right)$ & 10,0 & 4,5 & 4,0 & 8,0 \\
\hline Radiación solar recibida prom. $\left(\mathrm{W} \mathrm{m}^{-2}\right)$ & 182 & 350,0 & 263 & 165 \\
\hline Evaporación (mm) & 160 & 300,0 & 215 & 150 \\
\hline Parámetro de estratificación $(\phi)$ prom. $\left(\mathrm{J} \mathrm{m}^{-3}\right)$ & 1,68 & 35,39 & 29,80 & 0,17 \\
\hline Frecuencia Brunt-Väisälä promedio $\left(\mathrm{s}^{-1}\right)$ & $3,010^{-3}$ & $3,410^{-2}$ & $2,310^{-2}$ & $5,710^{-3}$ \\
\hline Frecuencia Brunt-Väisälä máxima $\left(\mathrm{s}^{-1}\right)$ & $1,010^{-2}$ & $4,4510^{-2}$ & $2,5510^{-2}$ & $6,510^{-3}$ \\
\hline Índice de surgencia inducido por el viento $\left(\mathrm{m}^{3} \mathrm{~s}-{ }^{1}\right)$ & No genera & 54,7 & 43,3 & No genera \\
\hline Esfuerzo del viento $\left(\mathrm{N} \mathrm{m}^{-2}\right)$ & $1,8210^{-1}$ & $3,6910^{-2}$ & $2,9110^{-2}$ & $1,1610^{-1}$ \\
\hline Gradiente horizontal de densidad $\left(\mathrm{kg} \mathrm{m}^{-3} \mathrm{~m}^{-1}\right)$ & $-8,8010^{-6}$ & $1,2610^{-5}$ & $1,4910^{-5}$ & $-8,1910^{-6}$ \\
\hline Estratificación por calentamiento $\left(\mathrm{W} \mathrm{m}^{-3}\right)$ & $5,5910^{-5}$ & $1,0710^{-4}$ & $8,0810^{-5}$ & $5,0710^{-5}$ \\
\hline Estratificación por flujo de densidad $\left(\mathrm{W} \mathrm{m}^{-3}\right)$ & $8,8010^{-7}$ & $1,9010^{-6}$ & $2,6410^{-6}$ & $7,6710^{-7}$ \\
\hline Estratificación por esfuerzo de marea $\left(\mathrm{W} \mathrm{m}^{-3}\right)$ & $-1.0310^{-5}$ & $1,6210^{-5}$ & $1,9910^{-5}$ & $-9,5910^{-6}$ \\
\hline Flujo impulsado por viento $\left(\mathrm{W} \mathrm{m}^{-3}\right)$ & $-1,9810^{-5}$ & $5,9910^{-6}$ & $5,6910^{-6}$ & $-1,1910^{-5}$ \\
\hline Mezcla por velocidad del viento $\left(\mathrm{W} \mathrm{m}^{-3}\right)$ & $7,6510^{-5}$ & $6,9810^{-6}$ & $4,9010^{-6}$ & $3,9210^{-5}$ \\
\hline Mezcla por corriente de marea $\left(\mathrm{W} \mathrm{m}^{-3}\right)$ & $3,3110^{-7}$ & $4,3710^{-7}$ & $4,9410^{-7}$ & $3,3110^{-7}$ \\
\hline
\end{tabular}


a)

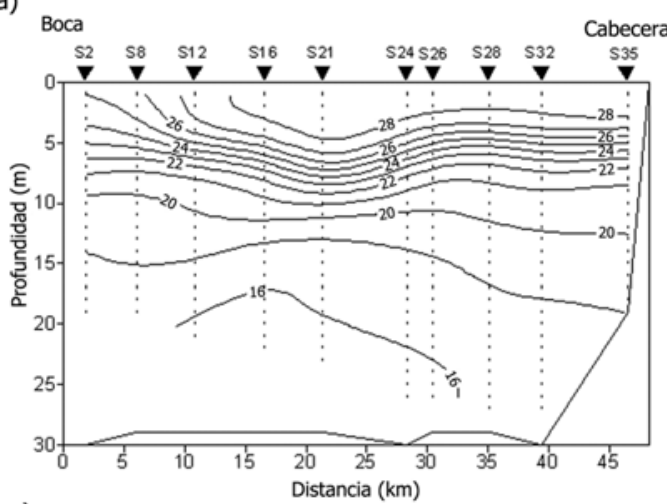

c)

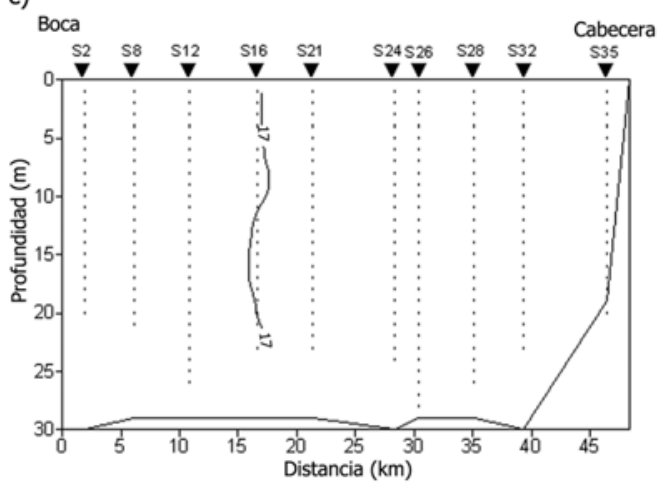

b)

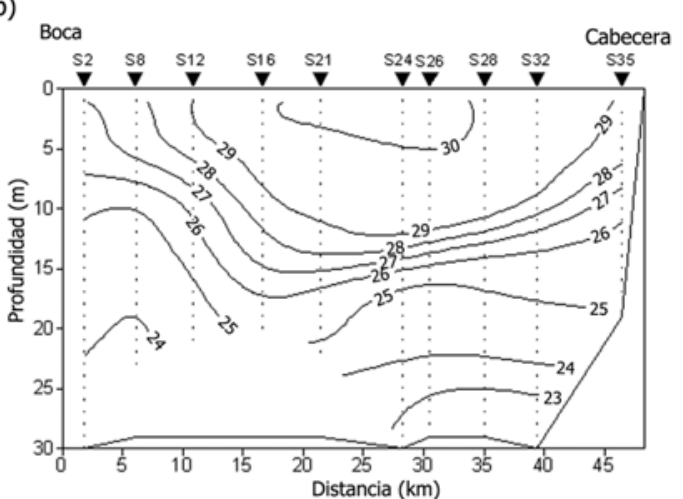

d)

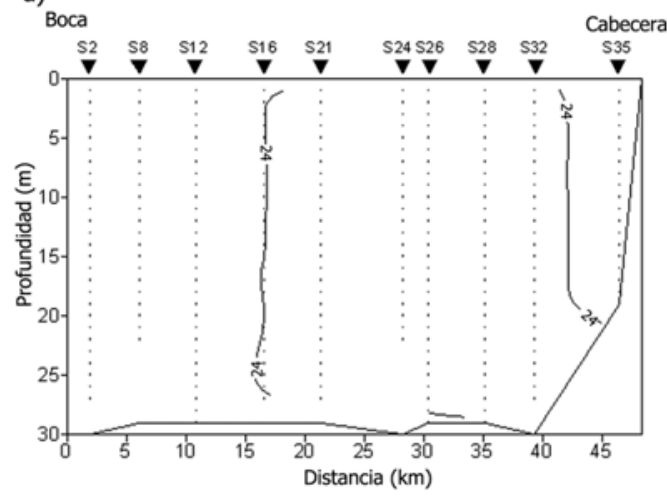

Figura 4. Sección longitudinal de temperatura. (a) Junio, (b) septiembre, (c) febrero y (d) noviembre de 1994 / Longitudinal section of temperature. (a) June, (b) September, (c) February and (d) November 1994

a) Costa Oeste

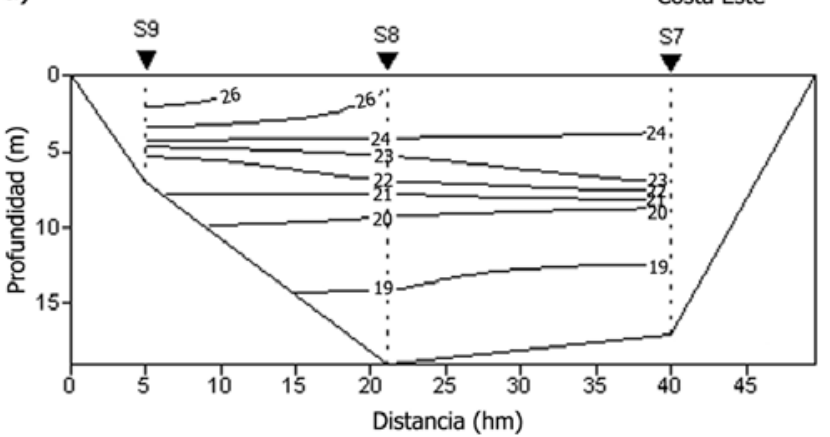

c) Costa Oeste

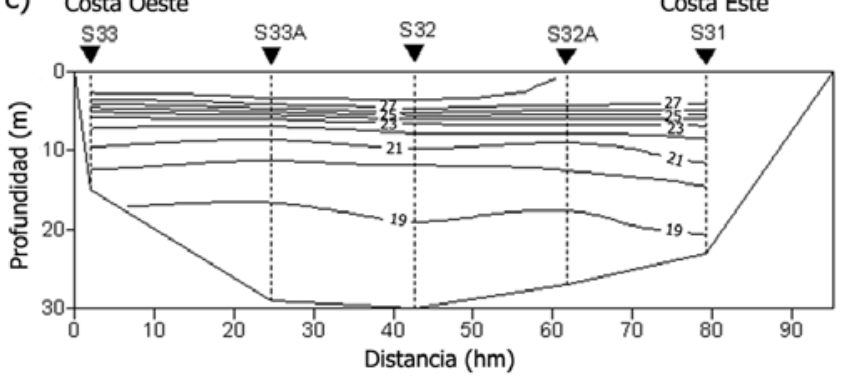

b) Costa Oeste

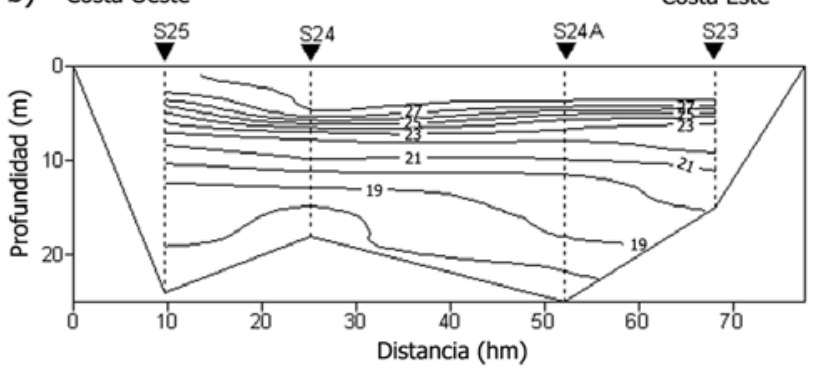

Figura 5. Sección transversal de temperatura. (a) Boca, (b) media y (c) cabeza de la bahía, junio de 1994 / Cross section of temperature. (a) Mouth, (b) middle, and (c) head of the bay, June 1994 

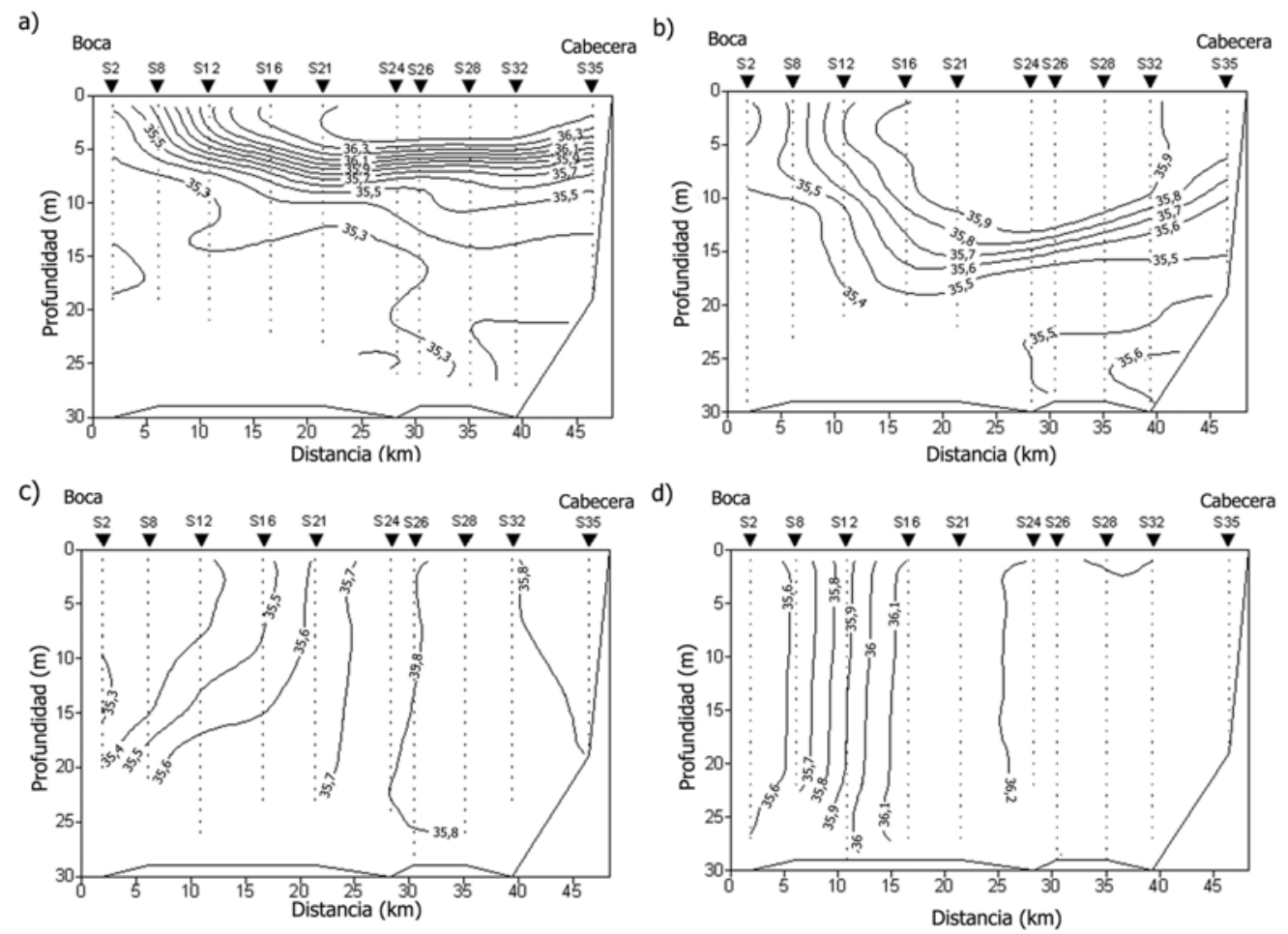

Figura 6. Sección longitudinal de salinidad. (a) Junio, (b) septiembre, (c) febrero y (d) noviembre de 1994 / Longitudinal section of salinity. (a) June, (b) September, (c) February and (d) November 1994
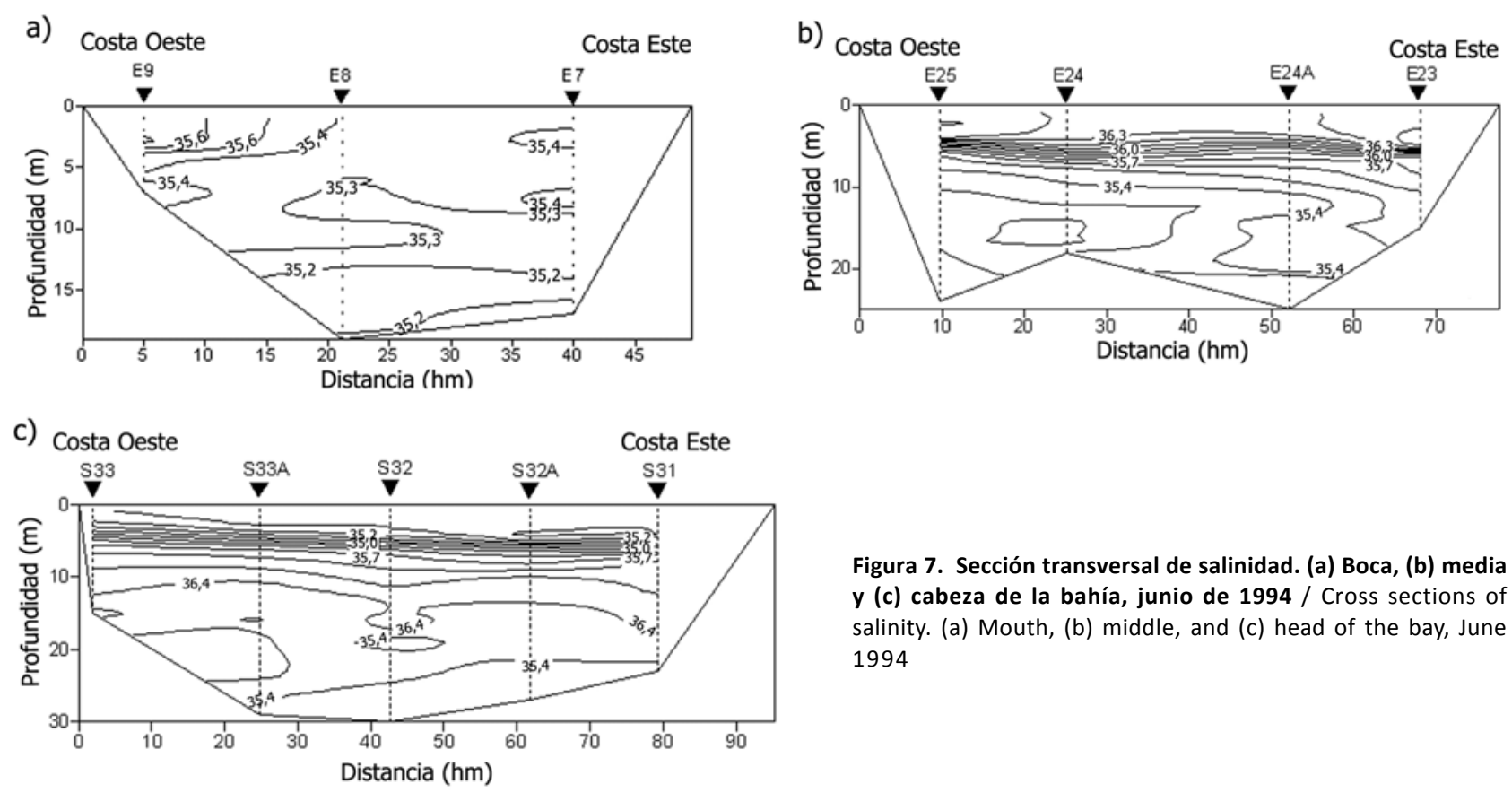

Figura 7. Sección transversal de salinidad. (a) Boca, (b) media y (c) cabeza de la bahía, junio de 1994 / Cross sections of salinity. (a) Mouth, (b) middle, and (c) head of the bay, June 1994 
Esta evolución de la temperatura y la salinidad en primavera (junio) de 1994, determinaron el comportamiento de la sigma-t en la bahía, con un promedio superficial de 23,41 $\mathrm{kg} \mathrm{m}^{-3}$, decreciendo rápidamente hacia el fondo, con una picnoclina bien desarrollada en los primeros $15 \mathrm{~m}$ de profundidad (Fig. 2c). En la sección longitudinal se apreció el agua superficial en la boca ligeramente más densa (> 23,4 $\mathrm{kg} \mathrm{m}^{-3}$ ) que en el resto de la bahía, la mayor estratificación se localizó en los primeros $10 \mathrm{~m}$ de profundidad y la isopicna de las $25,2 \mathrm{~kg} \mathrm{~m}^{-3}$, marcando el fondo de la picnoclina, con el afloramiento de las isopicnas de 23,4 y 23,6 $\mathrm{kg} \mathrm{m}^{-3}$, en la región de la boca entre las estaciones S2 y S16 (Fig. 8a).

En la distribución transversal de la densidad sobresale lo siguiente: en la sección de la boca la costa Oeste presentó la menor densidad, con el afloramiento de las isopicnas 23,4 y 23,6 $\mathrm{kg} \mathrm{m}^{-3}$, observándose cierta estratificación horizontal (Fig. 9a). En la sección media de la bahía la costa Este y la porción central de la sección registraron la menor densidad, con el afloramiento de la isopicna de $23,4 \mathrm{~kg} \mathrm{~m}^{-3}$ enfrente de la costa Oeste, se presentó la inclinación de las isopicnas hacia la costa Oeste entre los 2 y 10 m de profundidad (Fig. 9b). Entretanto en la sección de la cabeza la costa Este resultó ser la de mayor densidad, con el afloramiento de la isopicna de $23,4 \mathrm{~kg} \mathrm{~m}^{-3}$ en la parte central de la sección, la mayor estratificación se localizó entre los 3 y 15 de profundidad (Fig. 9c).
Los principales mecanismos de estratificación fueron por calentamiento con $1,0710^{-4} \mathrm{~W} \mathrm{~m}^{-3}$, el flujo de densidad de $1,9010^{-6} \mathrm{~W} \mathrm{~m}^{-3}$, esfuerzo de la marea de $1,6210^{-5} \mathrm{~W} \mathrm{~m}^{-3}$ y el flujo impulsado por el viento de $5,9910^{-6} \mathrm{~W} \mathrm{~m}^{-3}$, con un gradiente horizontal de densidad de $1,2610^{-5} \mathrm{~kg} \mathrm{~m}^{-3} \mathrm{~m}^{-1}$ en dirección a la cabecera (Tabla 1).

En el verano, la bahía alcanzó la máxima temperatura con un promedio superficial de $29,25^{\circ} \mathrm{C}$, observándose una capa de mezcla de $12 \mathrm{~m}$ en algunas estaciones de la porción central. La termoclina se profundizó y se extendió hasta los $25 \mathrm{~m}$ en la parte central de la bahía con un gradiente vertical de $0,49^{\circ} \mathrm{C} \mathrm{m}^{-1}$. El parámetro de estratificación ( $\phi$ ) promedio fue de 29,8 $\mathrm{J} \mathrm{m}^{-3}$ (Fig. 2a). En la frontera con el Golfo de California, se apreció nuevamente un gradiente horizontal superficial de temperatura de 2,12 $10^{-4}{ }^{\circ} \mathrm{C} \mathrm{m}^{-1}$ (Fig. 3b). En la sección longitudinal se observó la bahía muy cálida y estratificada, la temperatura se incrementó desde la boca $\left(27,0^{\circ} \mathrm{C}\right)$ hacia la parte central $\left(30,0^{\circ} \mathrm{C}\right)$, con la isoterma de las $24,0^{\circ} \mathrm{C}$ marcando el fondo de la termoclina. Se pudo apreciar en la región de la boca entre las estaciones S2 y S16 el afloramiento de las isotermas de 27,0 a $29,0^{\circ} \mathrm{C}$, mientras que en la cabecera afloró la isoterma de $29,0^{\circ} \mathrm{C}$ (Fig. 4b). El parámetro de estratificación $(\phi)$, también presentó una variación espacial, con el valor menor en la boca (20,75 J m ${ }^{-3}$, estación S2), se incrementó en la parte central (42,96 J m³ ${ }^{-3}$, estación S26) y disminuyó hacia la cabecera (37,44 $\mathrm{J} \mathrm{m}^{-3}$, estación S32).
Figura 8. Sección longitudinal de sigmat en $\mathrm{kgm}^{-3}$. (a) Junio, (b) septiembre, (c) febrero y (d) noviembre de 1994 / Longitudinal section of sigma-t en $\mathrm{kgm}^{-3}$. (a) June, (b) September, (c) February and (d) November 1994
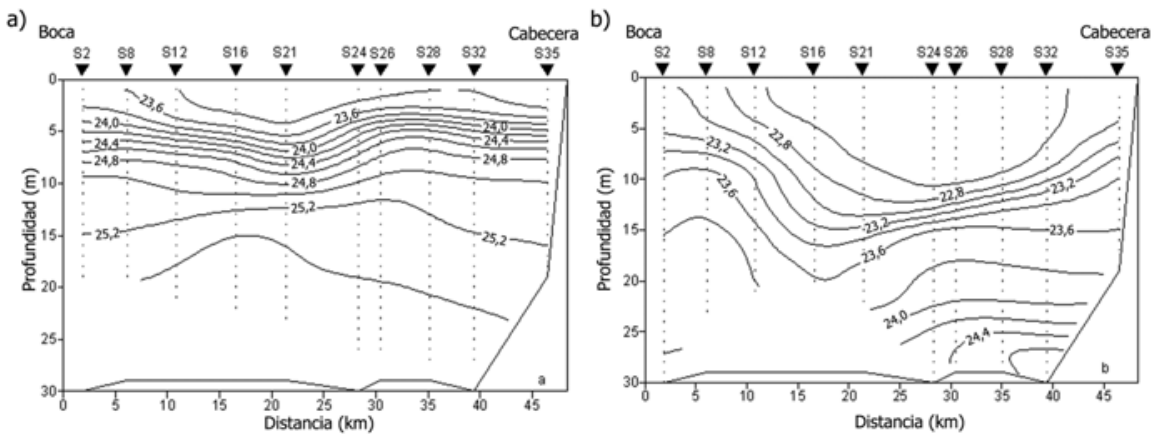

c)

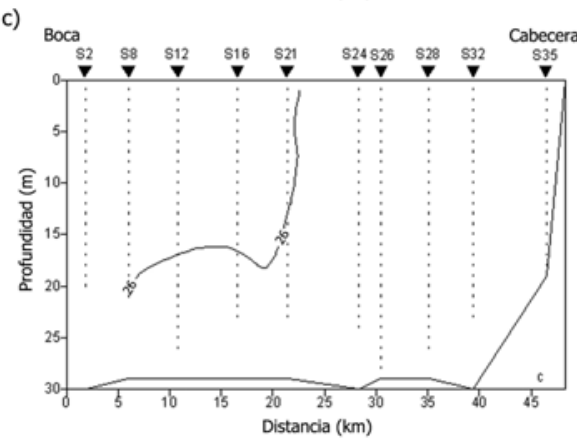

d)

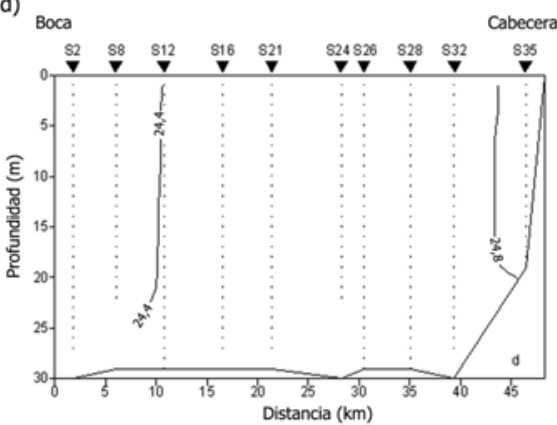



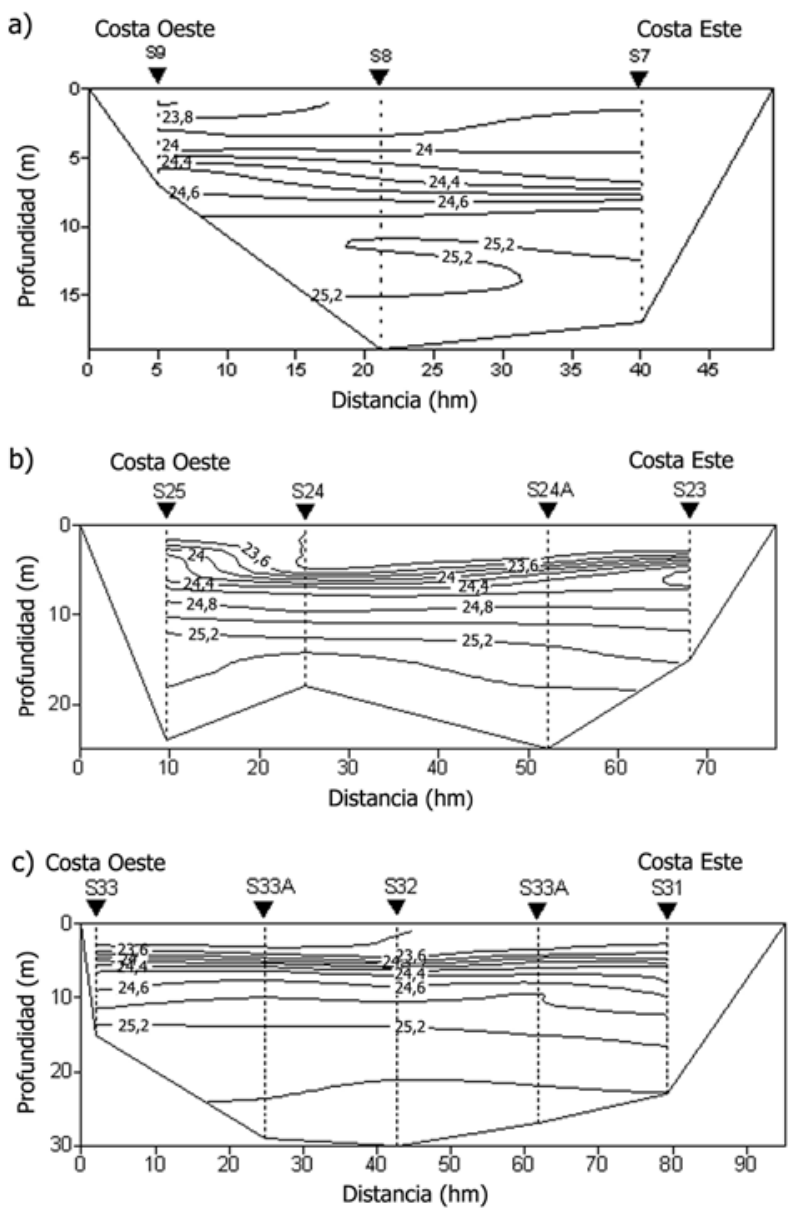

Figura 9. Sección transversal de sigma-t en $\mathrm{kg} \mathrm{m}^{-3}$. (a) Boca, (b) media y (c) cabeza de la bahía, junio de $\mathbf{1 9 9 4}$ / Cross section of sigma-t en $\mathrm{kg} \mathrm{m}^{-3}$. (a) Mouth, (b) middle, and (c) head of the bay, June 1994

Transversalmente, la distribución de temperatura en el verano mostró el comportamiento siguiente: en la sección de la boca la costa Este se presentó más cálida con el afloramiento de las isotermas 27,0 a $29,0^{\circ} \mathrm{C}$, con una débil estratificación horizontal; la costa Oeste presentó la menor temperatura de toda la bahía en este muestreo (Fig. 10a). En la sección media de la bahía, se registró la máxima temperatura $\left(30,0^{\circ} \mathrm{C}\right)$ de los 4 muestreos de 1994 , la temperatura fue homogénea de costa a costa, las isotermas se inclinan hacia la costa Oeste entre los $10 \mathrm{y}$ $16 \mathrm{~m}$ de profundidad, con estratificación horizontal (Fig. 10b). Entre tanto en la sección de la cabeza, se encontró una capa homogénea de $29,0^{\circ} \mathrm{C}$ de $9 \mathrm{~m}$ de profundidad de costa a costa, la estratificación se hundió y llegó hasta el fondo de la sección (Fig. 10c). La radiación solar promedio fue de $263 \mathrm{~W} \mathrm{~m}^{-2}$, con vientos del sureste de $4,0 \mathrm{~ms}^{-1} \mathrm{y}$ una evaporación de $215 \mathrm{~mm}$, con una frecuencia de Brunt-
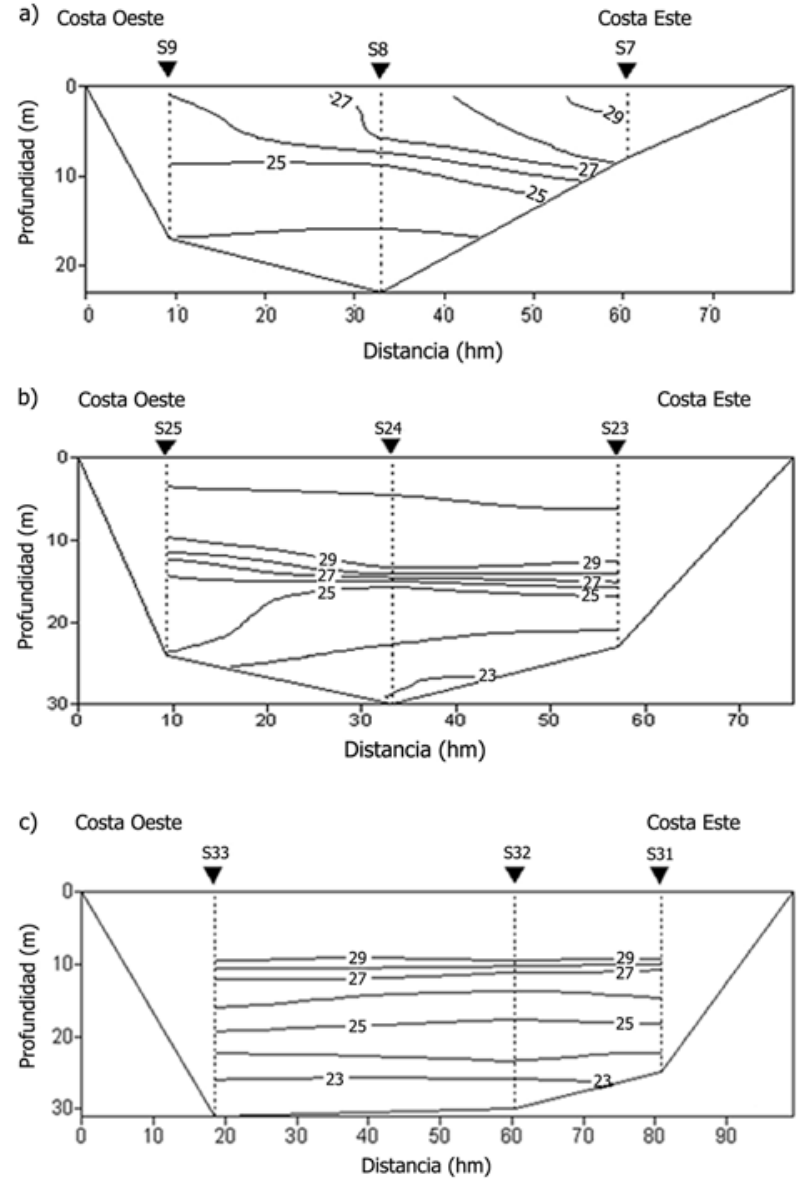

Figura 10. Sección transversal de temperatura. (a) Boca, (b) media y (c) cabeza de la bahía, septiembre de 1994 / Cross section of temperature. (a) Mouth, (b) middle, and (c) head of the bay, September 1994

Väisälä de 2,3 $10^{-2} \mathrm{~s}^{-1}$, con el valor máximo a los $15 \mathrm{~m}$ de profundidad $\left(2,5510^{-2} \mathrm{~s}^{-1}\right)$ y un índice de surgencia costera inducida por el viento de 43,3 $\mathrm{m}^{3} \mathrm{~s}^{-1}$ (Tabla 1).

En el verano de 1994, la salinidad presentó un promedio superficial de 35,81, la estratificación y la haloclina se debilitaron (Fig. 2b). En la frontera con el Golfo de California se apreció nuevamente un gradiente horizontal superficial de 6,42 $10^{-5} \mathrm{~m}^{-1}$ (Fig. 3d). En la sección longitudinal se pudo apreciar cierta estratificación vertical en la boca, con la menor salinidad $(35,4)$ alrededor de la estación S2 y el afloramiento de las isohalinas de 35,4 a 35,9. Mientras que la parte central mostró una estratificación horizontal y la mayor salinidad $(35,9)$, con una capa de mezcla de hasta $13 \mathrm{~m}$ de profundidad (estación S24), la haloclina se hundió en la parte central de la bahía con la isohalina de 35,5 marcando su límite profundo (Fig. 6b). 
Transversalmente, la bahía presentó la siguiente distribución de salinidad: en la sección de la boca se pudo apreciar la costa Oeste con la menor salinidad, mientras que en la costa Este se registraron 35,8, con el afloramiento de las isohalinas 35,4 a 35,8, al parecer con una intrusión de agua más salada desde la costa Este (Fig. 11a). En la sección media, la bahía se encontró separada verticalmente hasta los $12 \mathrm{~m}$ de profundidad, con la costa Este más salada con el afloramiento de la isohalina de 36,0, a mayor profundidad se encontró una estratificación horizontal, con la isohalina de 35,5 marcando el fondo de la haloclina (Fig. 11b). Entretanto, en la sección de la cabeza se registró una capa de mezcla de $10 \mathrm{~m}$ de profundidad, la isohalina de 35,9 marca el inicio de la haloclina, con la parte profunda estratificada horizontalmente (Fig. 11c).

En verano se registró en la bahía la menor densidad de los 4 muestreos de este año 1994, con una sigma-t superficial promedio de 22,6 $\mathrm{kg} \mathrm{m}^{-3}$, la picnoclina se debilitó y se hundió (Fig. 2c). Longitudinalmente, se
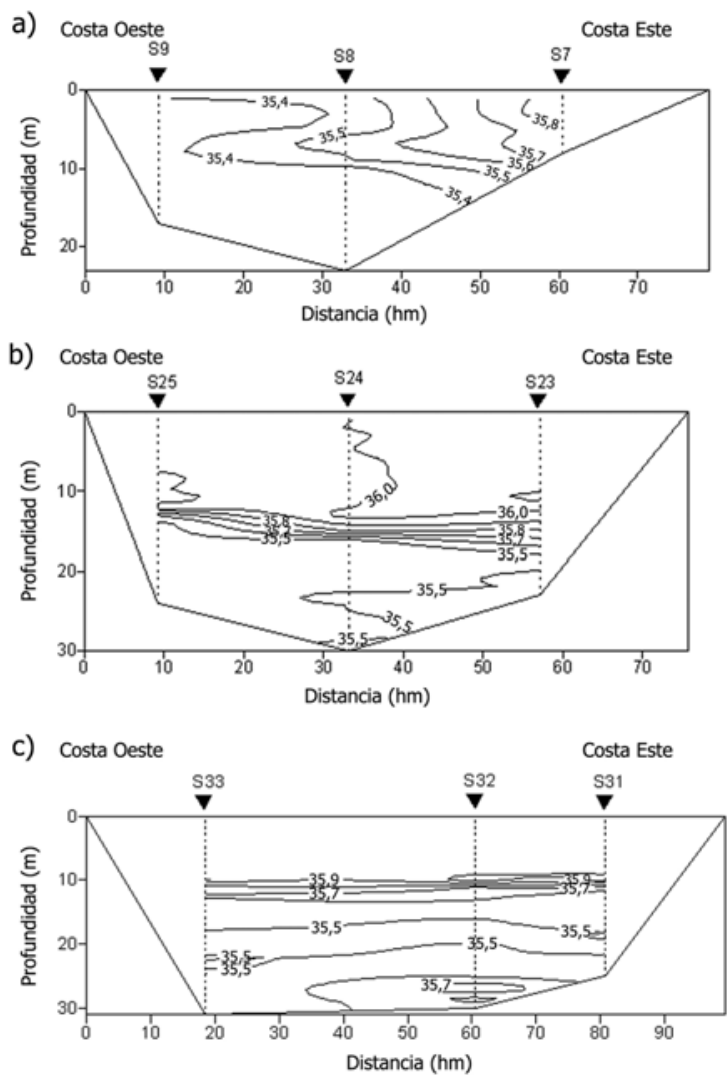

Figura 11. Sección transversal de salinidad. (a) Boca, (b) media y (c) cabeza de la bahía, septiembre de 1994 / Cross sections of salinity. (a) Mouth, (b) middle, and (c) head of the bay, September 1994 presentó en la boca el afloramiento de las isopicnas 23,0 a 22,6 $\mathrm{kg} \mathrm{m}^{-3}$ y en la cabecera la isopicna de $22,6 \mathrm{~kg} \mathrm{~m}^{-3}$. Mientras que en la porción central se registró una capa de mezcla de $10 \mathrm{~m}$ de profundidad, con la isopicna de 24,4 $\mathrm{kg} \mathrm{m}^{-3}$ limitando el fondo de la picnoclina (Fig. 8b).

Transversalmente, la distribución de sigma-t mostró las siguientes características: en la sección de la boca se apreció la costa Este con una menor densidad, con el afloramiento de las isopicnas de 22,60 a 23,2 $\mathrm{kg} \mathrm{m}^{-3}$, con una estratificación horizontal después de los $4 \mathrm{~m}$ de profundidad (Fig. 12a). En la sección media de la bahía se registró la costa Oeste más densa, con el afloramiento de la isopicna de 22,4 $\mathrm{kg} \mathrm{m}^{-3}$ en la costa Este, entre los 5 y 14 $\mathrm{m}$ de profundidad es clara la inclinación de las isopicnas hacia la costa Oeste (Fig. 12b). Entretanto, en la sección de la cabeza se registró una capa de mezcla de $8 \mathrm{~m}$ de profundidad en la costa Este, con la isopicna de $22,6 \mathrm{~kg}$ $\mathrm{m}^{-3}$ inclinada hacia la costa Oeste; la sección se apreció estratificada hasta el fondo (Fig. 12c).
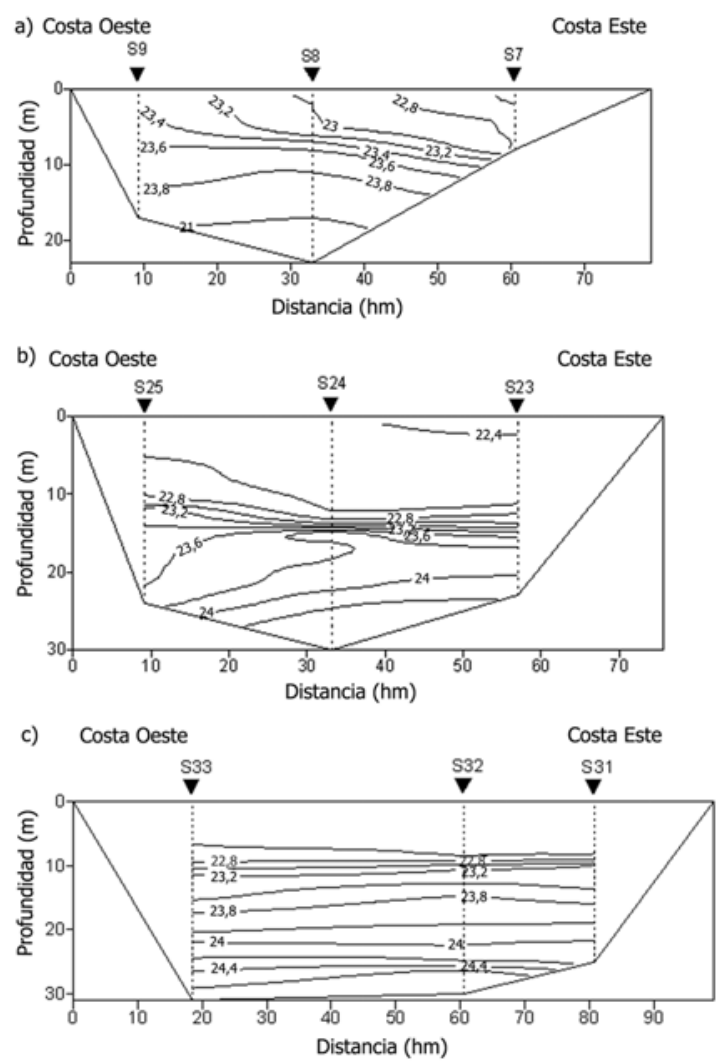

Figura 12. Sección transversal de sigma-t en $\mathrm{kg} \mathrm{m}^{-3}$. (a) Boca, (b) media y (c) cabeza de la bahía, septiembre de 1994 / Cross section of sigma-t en $\mathrm{kg} \mathrm{m}^{-3}$. (a) Mouth, (b) middle, and (c) head of the bay, September 1994 
En esta ocasión, los principales mecanismos de estratificación fueron por calentamiento $\left(8,0810^{-5} \mathrm{~W} \mathrm{~m}^{-3}\right)$, flujo de densidad $\left(2,6410^{-6} \mathrm{~W} \mathrm{~m}^{-3}\right)$, esfuerzo por marea $\left(1,9910^{-5} \mathrm{~W} \mathrm{~m}^{-3}\right)$ y el flujo impulsado por el viento (5,69 $\left.10^{-6} \mathrm{~W} \mathrm{~m}^{-3}\right)$, con un gradiente horizontal de densidad de $1,4910^{-5} \mathrm{~kg} \mathrm{~m}^{-3} \mathrm{~m}^{-1}$ en dirección a la cabecera (Tabla 1).

Las condiciones homogéneas y frías se registraron durante invierno (febrero) y otoño (noviembre), con las características que se describen a continuación. El perfil de temperatura mostró para el invierno de 1994, la temperatura superficial promedio mínima de este estudio $\left(17,07^{\circ} \mathrm{C}\right)$ (Fig. 2a). Longitudinalmente, la bahía estuvo muy fría y completamente homogénea (Fig. 4c), con una radiación solar promedio de $182 \mathrm{~W} \mathrm{~m}^{-2}$, una evaporación de $160 \mathrm{~mm}$ y vientos del noroeste de $10 \mathrm{~m} \mathrm{~s}^{-1}$ (Tabla 1).

En invierno 1994, el perfil de salinidad promedio reveló una bahía cuasi homogénea verticalmente, con la menor salinidad superficial promedio de 35,67, con una variación gradual mínima con la profundidad (Fig. 2b). En la sección longitudinal, se apreció una débil estratificación vertical en toda la bahía, con la salinidad menor en la boca $(35,3)$, que se incrementó gradualmente hacia la cabecera, registrándose la salinidad máxima $(35,8)$ entre la estación S26 y S32 (Fig. 6c).

Esta distribución de temperatura y salinidad en febrero, generaron el mayor sigma-t de los 4 muestreos en la bahía, con un promedio superficial de 26,03 $\mathrm{kg} \mathrm{m}^{-3}$ (Fig. 2c). Longitudinalmente, se registró una estructura homogénea, con una diferencia mínima de $0,2 \mathrm{~kg} \mathrm{~m}^{-3}$ entre la boca y la cabecera de la bahía, con el valor mínimo en la región de la boca (Fig. 8c). Los principales mecanismos de mezcla fueron la agitación por viento $\left(7,6510^{-5} \mathrm{~W} \mathrm{~m}^{-3}\right)$, mezcla por mareas $\left(3,3110^{-7} \mathrm{~W} \mathrm{~m}^{-3}\right)$ y el flujo impulsado por el viento $\left(1,9810^{-5} \mathrm{~W} \mathrm{~m}^{-3}\right)$, con un esfuerzo del viento de $1,8210^{-1} \mathrm{~N} \mathrm{~m}^{-2}$ (Tabla 1 ).

Mientras tanto en otoño (noviembre), nuevamente la bahía se encontró homogénea térmicamente, con una temperatura superficial promedio de $23,82^{\circ} \mathrm{C}$ (Fig. 2a). Longitudinalmente, se registró una temperatura de $24,0^{\circ} \mathrm{C}$ en la mayor parte de la bahía, mientras que en la boca se registraron $23,0^{\circ} \mathrm{C}$ (Fig. 4d). La radiación solar promedio fue de $165 \mathrm{~W} \mathrm{~m}^{-2}$, con vientos del noroeste de $8 \mathrm{~m} \mathrm{~s}^{-1} \mathrm{y}$ una evaporación de $150 \mathrm{~mm}$ (Tabla 1).

En la salinidad durante el otoño, desapareció la estratificación horizontal y se homogeniza verticalmente con un promedio superficial de 35,99, con un incremento mínimo gradual con la profundidad (Fig. 2b). En la sección longitudinal entre la boca y la cabecera se registró una estratificación vertical nuevamente, con la menor salinidad en la boca $(35,6)$, se incrementó gradualmente hacia la cabecera con el valor máximo de 36,2 después de la estación S24 (Fig. 6d).

La densidad durante el otoño se ha homogenizado verticalmente de nuevo con una sigma-t superficial promedio de $24,45 \mathrm{~kg} \mathrm{~m}^{-3}$ (Fig. 2c). Longitudinalmente, la menor densidad se registró en la región de la boca $(22,4$ $\mathrm{kg} \mathrm{m}^{-3}$ ) y se incrementó gradualmente hacia la cabecera (24,6 $\left.\mathrm{kg} \mathrm{m}^{-3}\right)$ (Fig. 8d). Los principales mecanismos de mezcla que generaron estas condiciones homogéneas fueron: la agitación por viento $\left(3,9210^{-5} \mathrm{~W} \mathrm{~m}^{-3}\right)$, la mezcla por mareas $\left(3,3110^{-7} \mathrm{~W} \mathrm{~m}^{-3}\right)$ y el flujo impulsado por el viento $\left(1,1910^{-5} \mathrm{~W} \mathrm{~m}^{-3}\right)$, con un esfuerzo del viento de $1,1610^{-1} \mathrm{~N} \mathrm{~m}^{-2}$ (Tabla 1$)$.

\section{Discusión}

Durante la condición estratificada y cálida, la disminución drástica de la temperatura, salinidad y densidad con la profundidad en junio de 1994, dio origen a una termoclina, haloclina y picnoclina intensas, causado principalmente por la radiación solar y el arribo de aguas del Golfo de California más frías y menos saladas, producto de la flotabilidad positiva originada por las surgencias costeras que se generan en estas épocas del año en la costa occidental del Golfo de California, debido a la acción de los vientos del sureste. Esto hace patente la influencia de las aguas del golfo en esta zona costera, y que coincide con lo reportado por Cheng et al. (2010) para verano de 2005, quienes muestrearon la temperatura de febrero a octubre con termistores, en una sección que cruza la bahía en la parte sur. También está parcialmente de acuerdo con lo reportado por Lechuga-Deveze et al. (2001), quienes muestrearon en la parte central de la bahía con botellas Van Dorn.

Según Molina-Cruz (1984), Salas (1966) y Marinone \& Lavín (1997) en verano, aparecen vientos del sureste cuando un sistema de baja presión se localiza en la porción noreste del Golfo de California (Roden 1958), y desplazan aire cálido y húmedo sobre el golfo. Las velocidades de estos vientos son generalmente débiles llegando hasta los $5 \mathrm{~m} \mathrm{~s}^{-1}$ y su humedad absoluta es alta (Badan-Dangon et al. 1991). La persistencia de estos vientos da origen a las surgencias costeras en la costa oriental de la península de Baja California.

La elevación y afloramiento de las isotermas, isohalinas e isopicnas en la sección transversal de la boca de la bahía, durante junio y septiembre de 1994, responde a la presencia de aguas frías, menos saladas y más densas, 
producto de las surgencias en este sector de la bahía y en la zona costera adyacente del Golfo de California, en esta época del año, originadas por los vientos del sureste que soplan paralelos a la costa Este de la península de Baja California. Esto viene a corroborar la deducción hecha por Cheng et al. (2010), con base en datos de termistores colocados en la porción sur de esta bahía, quienes afirman que al inicio del verano de 2005, la surgencia causó la intrusión de agua fría que bajo la temperatura de la columna de agua en Bahía Concepción.

La concavidad de las isotermas, isohalinas e isopicnas en la porción central de la bahía en septiembre de 1994, indican la posible existencia de un giro anticiclónico en la parte central de la bahía, condición que no había sido registrada por ningún autor a la fecha. Un comportamiento similar fue reportado por Salinas et al. (2003), para octubre de 1997 en una sección transversal en la parte central de Bahía de La Paz.

La variabilidad (longitudinal, transversal y con la profundidad) de temperatura, salinidad y densidad en Bahía Concepción durante las condiciones estratificadas y cálidas, presentó un mayor gradiente en junio de 1994, que en septiembre del mismo año, debido al parecer principalmente a una mayor radiación solar recibida, un mayor esfuerzo del viento, una mayor estratificación por calentamiento y a la participación de la surgencia en el área de la boca de la bahía y la zona adyacente del Golfo de California.

La homogeneidad de temperatura, salinidad y sigma-t registradas en la bahía durante el invierno y otoño de 1994, fueron producidas por la menor radiación solar recibida durante estas épocas del año, el flujo impulsado por el viento y el proceso de mezcla producto de los fuertes vientos del noroeste. Estas condiciones, aunadas al intercambio con el Golfo de California de aguas con menor salinidad, dieron origen a la estructura vertical de la salinidad, con un incremento gradual desde la boca hacia la cabecera. Según Cheng et al. (2010), durante invierno y otoño los vientos del noroeste dominan y causan hundimiento en la costa oriental de la península de Baja California, ellos registraron los vientos en la boca y la cabecera de la bahía de febrero a octubre de 2005. Además, coincide en parte con lo reportado por Palomares-García et al. (2006), quienes muestrearon bisemanalmente temperatura y salinidad en una estación localizada en la parte central de la bahía durante un año.

Las condiciones meteorológicas de otoño e invierno en Bahía Concepción son originadas a mayor escala en el Golfo de California por la influencia de los vientos estacionales que, durante estas épocas del año, son canalizados a lo largo de su eje longitudinal entre las cadenas montañosas situadas en la península de Baja California y el relieve continentales. Estos patrones de vientos fueron descritos y discutido por Molina-Cruz (1984), Salas (1996) y Marinone \& Lavín (1997), quienes señalan que tales vientos están sujetos a fluctuaciones estacionales como consecuencia de la migración del sistema de baja presión localizado sobre el occidente de México. Cuando el sistema de baja presión se encuentra en el lado oriental del golfo, que es la mayor parte del año, se presentan vientos del noroeste secos y fríos sobre el golfo, con velocidades de $10-12 \mathrm{~m} \mathrm{~s}^{-1}$ que producen hundimientos en la costa oriental de la península de Baja California.

La variabilidad temporal de los valores de la frecuencia de Brunt-Väisälä (N) no escapó de la señal estacional. Una combinación de baja actividad eólica y la intensa radiación solar recibida durante primavera y verano del año de 1994, contribuyó a obstaculizar el proceso de mezcla favoreciendo la estratificación y la ocurrencia de valores altos de $\mathrm{N}$ para este periodo del año. De manera opuesta, la intensa actividad eólica local y la baja radiación solar recibida durante otoño e invierno del año de 1994 arrojó como resultado la mezcla y homogenización de la columna de agua y valores bajos de $\mathrm{N}$.

El mecanismo de estratificación en la bahía fue generado principalmente por el calentamiento, las surgencias costeras y el flujo impulsado por el gradiente de densidad, mientras que el proceso de mezcla tuvo su origen esencialmente en los vientos y el enfriamiento.

Es importante señalar que, el frente termohalino en la boca de la bahía durante la primavera y verano, fue generado por la importante radiación solar en la región y las surgencias generadas por los vientos del sureste, en la comunicación con el Golfo de California.

La variabilidad de la temperatura, salinidad, densidad y estratificación en la bahía tienen un comportamiento que va de un medio homogéneo y frío (noviembre a febrero) a un ambiente estratificado y cálido (junio a septiembre), tal vez separados por dos períodos corto de transición.

Finalmente, se concluye que la distribución de temperaturas, salinidad y densidad en Bahía Concepción difieren espacial y estacionalmente, producto de la radiación solar, estratificación por calentamiento, mezcla por viento, flujo impulsado por el viento y las surgencias que se presentan en la boca, frontera con el Golfo de California. 


\section{Agradecimientos}

Los autores agradecen al Instituto Politécnico Nacional quien a través de la Secretaria de Posgrado e Investigación, financiaron las expediciones y al CICIMAR por proporcionar la embarcación, instalaciones y personal para la obtención de los datos, así como al Servicio Meteorológico Nacional de México quien proporcionó los datos de radiación solar y a la Comisión Nacional del Agua por los datos de vientos y evaporación.

\section{LITERATURA CITADA}

Badan-Dangon A, CE Dorman, MA Merrifield \& CD Winant. 1991. The lower atmosphere over the Gulf of California. Journal of Geophysical Research 96(C9): 1687716896.

Cheng P, A Valle-Levinson, CD Winant, ALS Ponte, G Gutierrez-Velasco \& KB Winters. 2010. Upwellingenhanced seasonal stratification in a semiarid bay. Continental Shelf Research 30: 1241-1249.

Félix-Pico EF. 1987. Scallop fishing in Bahia Concepcion, Baja California Sur, Mexico, 65 pp. International. Pectinid Workshop, Menai Bridge.

Gilmartin M \& N Revelante. 1978. The phytoplankton characteristics of the barrier Island Lagoons of he Gulf of California. Estuarine Coastal and Shelf Science 7: 29-47.

Lechuga-Deveze CH \& ML Morquecho-Escamilla. 1998. Early spring potentially harmful phytoplankton in Bahia Concepcion, Gulf of California. Bulletin of Marine Science 63: 503-512.

Lechuga-Deveze CH, A Reyes-Salinas \& ML MorquechoEscamilla. 2001. Anoxia in a coastal bay: case study of a seasonal event. Revista de Biología Tropical 49: 525-534.

Marinone SG \& MF Lavín. 1997. Mareas y corrientes residuales en el Golfo de California. En: Lavín MF (ed). Contribuciones a la oceanografía física en México. Monografía 3: 113-139. Unión Geofísica Mexicana, Ensenada.

Martínez-López A \& I Gárate-Lizárraga. 1994. Cantidad y calidad de la materia orgánica particulada en Bahía Concepción en la temporada de reproducción de la almeja catarina Argopecten circularis (Sowerby, 1835). Ciencias Marinas 20(3): 301-320.

Mateo-Cid LE, I Sánchez-Rodríguez, YE RodríguezMontesinos, M Casas-Valdéz. 1993. Estudio florístico de las algas marinas bentónicas en Bahía Concepción, B.C.S., México. Ciencias Marinas 19: 41-60.

Molina-Cruz A. 1984. Evolución oceanográfica de la Boca del Golfo de California. Anales del Instituto de Ciencias del Mar y Limnología, Universidad Nacional Autónoma de México 13(2): 95-120.
Obeso-Nieblas M. 2003. Variabilidad espacio-temporal de las condiciones oceanográficas de la Bahía de La Paz, BCS, México. Tesis Doctoral, Centro Interdisciplinario de Ciencias Marinas, IPN, La Paz, Baja California Sur, 337 pp.

Obeso-Nieblas M, MA Alatorre-Mendieta \& AR JiménezIllescas. 1996. Modelación de la marea en Bahía Concepción, BCS, México. Oceánides 11: 1-8.

Pacanowski RC \& SGH Philander. 1981. Parameterization of vertical mixing in numerical models of tropical oceans. Journal of Physical Oceanography 11: 1143-1151.

Palomares-García R, A Martínez-López \& I Gárate Lizárraga. 2002. Plankton community changes in Bahia Concepcion, México. Oceánides 17(2): 113-128.

Palomares-García R, JJ Bustillo-Guzmán \& D López Cortés. 2006. Pigment-specific rates of phytoplankton growth and microzooplankton grazing in a subtropical lagoon. Journal of Plankton Research 28: 1217-1232.

Plata L \& A Filonov. 2007. Marea interna en la parte noroeste de la Bahía de Banderas, México. Ciencias Marinas 33(2): 197-215.

Roden GI. 1958. Oceanographic and meteorological aspects of the Gulf of California. Pacific Science 12: 21-45.

Rodríguez-Romero J, LA Abitia-Cárdenas, F GalvánMagaña \& H Chávez-Ramos. 1994. Composition, abundance and specific richness of fishes from Concepcion Bay, Baja California Sur, Mexico. Ciencias Marinas 20: 321-350.

Salas PJ de J. 1996. El intercambio de agua sobre los umbrales del Golfo de California. Tesis de Maestría, Centro de Investigación Científica y de Educación Superior de Ensenada, Baja California, México, 65 pp.

Salinas-González F, O Zaytsev \& M Vyacheslav. 2003. Formación de la estructura termohalina del agua en la Bahía de La Paz de verano a otoño. Ciencias Marinas 29: 51-65.

Silva N \& A Valdenegro. 2003. Evolución de un evento de surgencia frente a punta Curaumilla, Valparaíso. Investigaciones Marinas 31(2): 73-89.

Simpson JH. 1981. The shelf sea front: Implication of their existence and behavior. Philosophical Transactions of the Royal Society of London 302: 531-546.

Simpson JH, J Brown, J Matthews \& G Allen. 1990. Tidal straining, density currents, and stirring in the control of estuarine stratification. Estuaries 13(2): 125-132.

UNESCO. 1991. Processing of Oceanographic Station Data, 138 pp. JPOTS Editorial Panel, Paris.

Villalejo-Fuerte M \& RI Ochoa-Báez. 1993. El ciclo reproductivo de la almeja Catarina Argopecten circularis (Sowerby, 1835), en relación con temperatura y fotoperíodo, en Bahía Concepción, B.C.S., México. Ciencias Marinas 19: 181-202.

Recibido el 27 de noviembre de 2011 y aceptado el 5 de noviembre de 2012

Editor Asociado: Mauricio Landaeta D. 